\title{
A Rare Case of Emphysematous Urethritis
}

\author{
Sisi Chen ${ }^{1}$, Sybghat Rahim ${ }^{2}$, Soumendra Nath Datta ${ }^{1 *}$ \\ ${ }^{1}$ Colchester Hospital University NHS Foundation Trust, Turner Road, Colchester, England \\ ${ }^{2}$ Cedar House GP practice, 14 Huntingdon Street, St. Neots, Cambridgeshire, England
}

Received: June 16, 2016; Accepted: June 18, 2016; Published: June 25, 2016

*Corresponding author: Soumendra Nath Datta, Colchester Hospital University NHS Foundation Trust, Turner Road, Colchester, Essex CO4 5JL, England, Tel: +07965 803351; E-mail: snd999@gmail.com

\begin{abstract}
Emphysematous urethritis can present with non-specific fever and generalised abdominal pain with no associated skin changes. Although rare, a suspicion for this diagnosis should be raised for anyone presenting with signs of septic shock without any signs of focal infection. Cross sectional imaging and surgical intervention should be sought early.
\end{abstract}

Keywords: Infection/Inflammation; Imaging; Urethral Reconstruction

\section{The Case}

\section{Clinical history}

A 49-year-old Caucasian man presented to the Emergency Department with a 7-day history of worsening diarrhoea and vomiting, intermittent chest pain and feeling generally unwell. He had a known history of chronic alcoholism with previous admissions for alcohol-related falls and withdrawal seizures; epilepsy post road traffic accident; and recurrent sinusitis.

\section{Physical examination}

He weighed 45 kilograms on admission and although unkempt and cache tic on initial inspection, he was alert and orientated. Initial observations showed blood pressure 73/40, heart rate 110 , respiratory rate 30 and oxygen saturation $99 \%$ on high flow oxygen. Auscultation of the chest revealed reduced air entry bilaterally with coarse inspiratory crepitations; heart sounds were normal. The abdomen was soft but diffusely tender, bowels sounds were normal. Digital rectal examination revealed a mildly tender prostate. Neurological examination was normal.

\section{Diagnostic studies}

Computer Tomography (CT) of the chest showed bilateral pleural effusions extending into the apical regions, bibasilar compressive atelectasis, as well as right upper and lower lobe consolidation. CT of the abdomen and pelvis showed a $4 \mathrm{~cm}$ fluid collection surrounding the membranous urethra, and there was also a linear fluid collection seen surrounding the penile urethra with multiple small locules of gas within the corpora spongiosum in keeping with necrotic tissue. The liver, spleen, adrenals and kidneys had unremarkable appearances. Septic shock secondary to urethral and bulbous necrotising infection was diagnosed. Hepatitis and HIV screening were negative, as were urinary cultures.

\section{Intervention}

The patient was catheterised via the urethra, resuscitated with intravenous fluids, and given broad-spectrum antibiotics co-amoxiclav and gentamicin. He was admitted to the intensive care unit and received Norepinephrine infusion to support his blood pressure.

The patient underwent emergency surgical exploration of his perineum, excision of urethra and insertion of a suprapubic catheter by expanding the bladder with the urethral catheter that was placed at admission. The pus-filled collection surrounding the urethra was drained and all necrotic tissue derided. Samples of tissue were sent to the Microbiology Laboratory, isolating Streptococcus miller and Bactericides fragilis. Histology showed extensive infarction of the corpus spongiosum and overlying urethral urothelium; there was associated dense acute inflammation with abscess formation. This was consistent with necrotising fasciitis. The patient continued treatment with co-amoxiclav and metronidazole. A further exploration of the perineal wound was performed 48 hours after the initial surgery. At second operation, the wound was clean with no further signs of necrosis or infection. A repeat CT abdomen was performed on Day 5 following admission which showed 3 newly developed hypo-dense splenic lesions (in keeping with infarction), as well as a large infarcted area in the upper pole and mid pole cortex of the left kidney. This was thought likely to be secondary to sepsis.

\section{Outcome}

Clinically the patient had a slow recovery but was successfully extubated on Day 6 post admission. He had extensive care input from dieticians and physiotherapists. The long-term plan is for urethral reconstruction once he recovers fully from the acute septic episode.

\section{Discussion}

Although urinary cultures have been negative in this case, the most likely aetiology is a similar picture to Fourniere's gangrene, 
i.e. spread from a urinary focus proximally or perineal infection. The history of diarrhoea prior to admission in this case could indicate a primary anorectal source whereby organisms migrated towards the genitourinary tract.

Gas-Forming Urinary Tract Infections (UTIs) also known as 'emphysematous UTIs' have a high mortality rate (70-90\%)[1], although they form a very small percentage of UTIs overall. ${ }^{1}$ The pathology of gas-forming UTIs involvesrapidly progressive necrotising inflammation caused by gas-forming organisms within the urinary tract, i.e. necrotising fasciitis [2]. The first case was described in 1671.

The symptoms for necrotising fasciitis are non-specific and notoriously difficult to diagnose, the incidence overall is approximately 500 cases per year in the UK [1]. The high mortality rates reflect difficulty and delay in diagnosis due to the rarity of these diseases and their subtle initial signs [2]. Often patients present with pain and fever, followed possibly by cellulitic skin changes, which often mimic haematoma, bursitis, phlebitis, sciatica, cellulites, septic arthritis or deep venous thrombosis [2]. The typical textbook description of haemorrhagic bullae, crepitus and skin necrosis often do not occur until Day 5 or later [1]. The greatest diagnostic difficulty comes when a patient presents with pain without fever. This pain is secondary to a combination of tissue necrosis as well as infarction of nerve tissue due to thrombosis of the associated blood vessels. Often the pain reported by the patient exceeds what can be identified clinically; this should raise high suspicion for a diagnosis of necrotising fasciitis [1].

A range of emphysematous UTIs have been reported which affect different parts of the urinary tract - Emphysematous pyelonephritis, emphysematous pyelitis, emphysematous cystitis and emphysematous prostatitis. The characteristics of each of these emphysematous UTIs are summarised in table 1 . To date, no cases of emphysematous urethritis have been reported to the best of our knowledge.
Predisposing factors to all emphysematous UTIs include Diabetes Mellitus (DM), immunosuppressant, cirrhotic liver disease, alcoholism, recent urethral instrumentation and (specifically for emphysematous pyelitis and cystitis) urinary obstruction [2].

Immunosuppressant is a key predisposing factor in emphysematous urinary tract infections [1]. Without doubt in the current case alcoholism was the key mediator of immunosuppressant. Acute alcohol binges result in impaired production of pro-inflammatory cytokines involved in regulation of immune cells, as well as impairing macrophage function and over time altering the response of the hypothalamic-pituitaryadrenal axis to acute immune challenges [3]. It is likely in the current case that cachexia and poor nutrition also exacerbated the extent of the infection.

Emphysematous pyelonephritis is the most commonly reported emphysematous UTI. It is characterised by gas formation in the renal parenchyma, perinephritic space or collecting system [2]. $90 \%$ of patients reported with this condition so far have been diabetic [2]. Emphysematous pyelonephritis is classified as either Type 1 or 2 [1]. Type 1 is progressive fulminant infection with widespread necrosis, intravascular thrombosis, and microabscess and gas formation. The mortality rate is 70-90\% [2], and with those that survive the kidney becomes non-functioning [2]. Type 2 has a more indolent course with renal and perirenal fluid collections with loculated gas. The mortality rate in type 2 is approximately $20 \%$ [2]. The causative organisms isolated to date include Escherichia coli (68-71\%), Klebsiella spp (9-15\%), Enterobacter aerogenes (10\%), Proteus spp (5\%), Pseudomonas spp and rarely yeast. In cases with abscess formation, the mainstay of treatment should be a combination of abscess drainage and antimicrobial treatment [2]. Nephrectomy may need to be considered at a later stage.

Emphysematous prostatitis involves gas and exudates formation in the prostate gland. It is also known as

Table 1:

\section{Emphysematous}

\begin{tabular}{|c|c|c|c|c|}
\hline & Pyelonephritis & Pyelitis & Cystitis & Prostatic \\
\hline $\begin{array}{l}\text { Path gnomonic symptoms and } \\
\text { signs }\end{array}$ & None & None & Pneumouria & None \\
\hline Causative organisms & $\begin{array}{l}\text { E. coli, } \\
\text { Klebsiella spp, } \\
\text { Enterobacter aerogenes, } \\
\text { Proteus spp, Pseudomonas } \\
\text { spp, } \\
\text { rarely Candida }\end{array}$ & $\begin{array}{l}\text { similar to } \\
\text { emphysematous } \\
\text { pyelonephritis }\end{array}$ & $\begin{array}{l}\text { E. coli, } \\
\text { Enterobacter spp, Klebsiella } \\
\text { spp, } \\
\text { Proteus spp, Staphylococcus } \\
\text { spp, Streptococcus spp, } \\
\text { Nocardia, } \\
\text { Clostridium spp, } \\
\text { rarely Candida }\end{array}$ & $\begin{array}{l}\text { E.coli, } \\
\text { Klebsiella pneumoniae, } \\
\text { Proteus mirabilis, } \\
\text { Citrobacter and yeasts }\end{array}$ \\
\hline Mortality rates & $\begin{array}{l}\text { Type 1: } 70-90 \% \text {; } \\
\text { Type 2: } 20 \%\end{array}$ & $20 \%$ & $20 \%$ & $25 \%$ \\
\hline Management & $\begin{array}{l}\text { abscess drainage and } \\
\text { microbial therapy, rarely } \\
\text { nephrectomy }\end{array}$ & antimicrobial therapy & $\begin{array}{l}\text { bladder drainage and } \\
\text { antimicrobial therapy }\end{array}$ & $\begin{array}{l}\text { abscess drainage and microbial } \\
\text { therapy }\end{array}$ \\
\hline
\end{tabular}



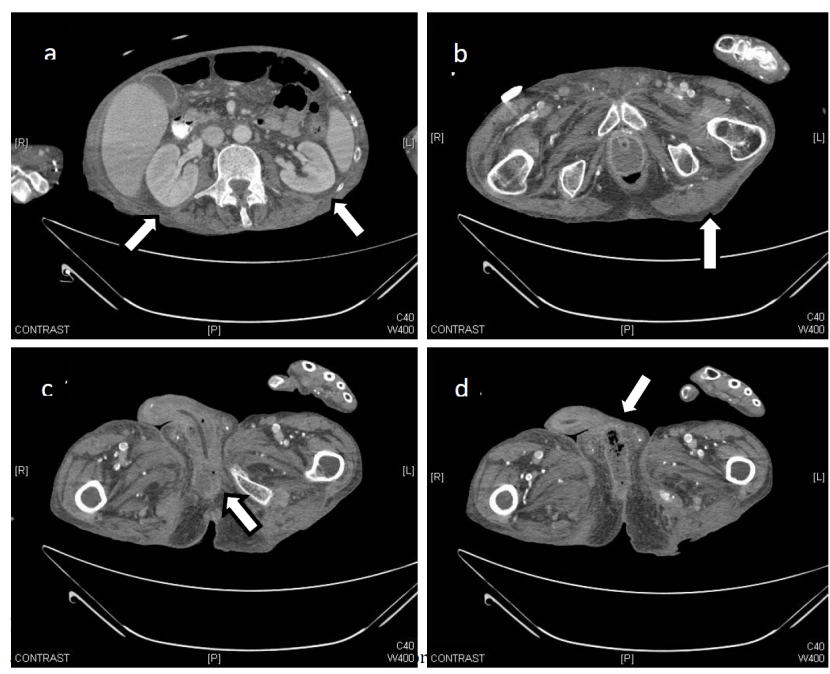

Figure 1: a) Normal kidneys. b) Normal prostate with posterior gas containing abscess. c) \& d) Gas containing fluid in per-urethral tissue.

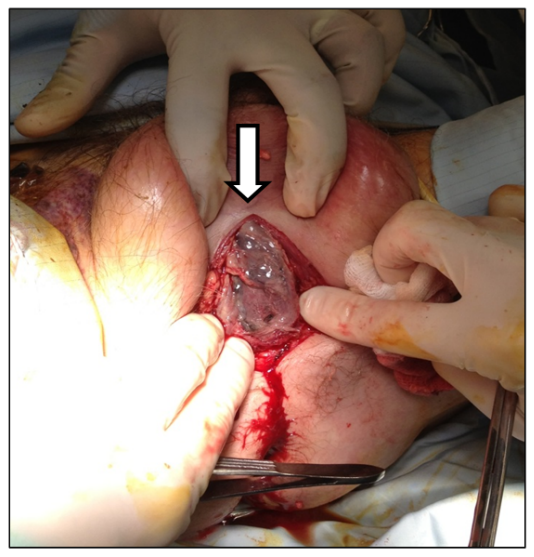

Figure 2: Intra-operative photograph of necrotic urethral tissue.
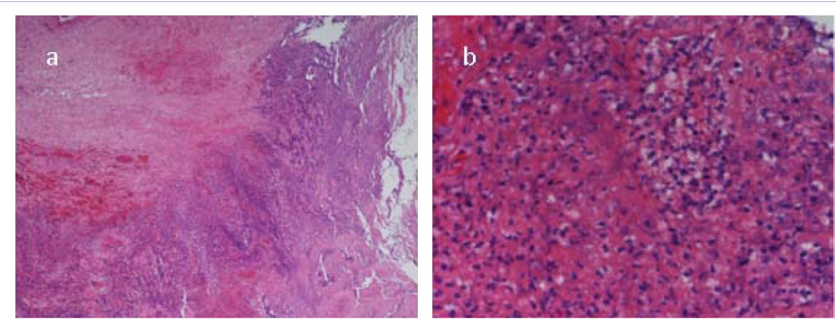

Figure 3: Photomicrographs of the debrided tissue, stained with hematoxylin and eosin. a) Low power image (x 100) showing extensive infarction of the corpus spongiosum with infarction of overlying urethral urothelium. b) High power image ( $\mathrm{x} 400$ ) showing dense acute inflammatory cells within the infarcted tissue.

Emphysematous Prostatic Abscess (EPA) in the literature, and is the closest pathological process which our current case can draw parallel comparisons to. 12 cases of EPA have been reported to date $^{4}$, with the first case reported in 1983. It has a high mortality rate of $25 \%$, and 10 out of 12 cases occurred in patients with
DM. It is difficult to differentiate EPA to acute prostatitis as there are no pathogmonic symptoms for EPA. Most frequently patients present with symptoms of dysuria, urinary frequency and urgency, as well as pyrexia, acute retention and perineal pain [4]. Diagnosis requires a high clinical suspicion. A digital rectal examination often reveals localized tenderness in a soft and fluctuant prostate Accurate and timely imaging is essential.

In our current case, there were no clear urinary symptoms and the patient had non-specific abdominal symptoms. Imaging was crucial to raise suspicion for the diagnosis. A screening CT was requested due to a lack of focus for infection in a patient with severe sepsis. CT is considered the most sensitive and specific imaging modality at present for necrotising fasciitis, as plain film x-ray has low sensitivity and specificity. In emphysematous pyelonephritis, a plain film has a yield of less than 50\% [5]. In cases of EPA, intravenous pyelography has revealed non-specific findings and would not be helpful in our case to demonstrate peri-urethral collections [5]. CT is therefore the best imaging modality at present for both diagnosis and monitoring response to treatment [5]. It can identify clearly the location of gas formation, differentiating between emphysematous pyelonephritis, perinephritic, pyelitis, cystitis and prostatitis and as in this case, urethritis [5]. Magnetic resonance imaging is also sensitive but there is limited evidence comparing this modality with CT scanning. In addition, it is often neither available nor feasible in a timely manner.

Our long-term challenge will be whether the lower urinary tract can be reconstructed. The majority of the anterior urethra has been excised and any reconstruction will need extensive tissue flaps or grafts, but only after complete recovery of the patient. The alternative is perineal urethrostomy or long term suprapubic catheterisation.

\section{Conclusion}

This case study reports a unique case of emphysematous urethritis (necrotising fasciitis). This rapidly progressive soft tissue infection requires a high index of suspicion together with prompt CT cross-sectional imaging. Our management was based around aggressive fluid resuscitation, antibiotic therapy and surgical debridement and drainage. To the best of our knowledge, this article is the first to report a case of this nature, particularly as this case described a relatively young patient without a common underlying co-morbidity such as diabetes mellitus.

\section{References}

1. Mokabberi R, Ravakhah K. Emphysematous urinary tract infections: diagnosis, treatment and survival (case review series). Am J Med Sci. 2007;333(2):111-6

2. Hasham S, Matteucci P, Stanley PRW. Necrotising fasciitis. BMJ. 2005;330(7495):830-3.

3. Silva SM, Madeira MD. Effects of chronic alcohol consumption and withdrawal on the response of the male and female hypothalamicpituitary-adrenal axis to acute immune stress. Brain Res. 2012;1444:27-37. doi: 10.1016/j.brainres.2012.01.013.

4. Wen SC, Juan YS, Wang CJ, Chang KO, Ming-Chen PS, Jung-Tsung S, et 
al. Emphysematous prostatic abscess: case series study and review. International Journal of Infectious Diseases. 2012;e344-349.
5. Bohlman ME, Sweren BS, Khazan R, Minkin SD, Goldman SM, Fishman EK, et al Emphysematous pyelitis and emphysematous pyelonephritis characterized by computerized tomography. South Med J. 1991;84(12):1438-43. 